\title{
Statins and the risk of intracerebral haemorrhage in patients with stroke: systematic review and meta- analysis
}

\author{
Oliver Jonathan Ziff, ${ }^{1,2}$ Gargi Banerjee, ${ }^{1}$ Gareth Ambler, ${ }^{3}$ David J Werring ${ }^{1,2}$
}

\begin{abstract}
- Additional material is published online only. To view please visit the journal online (http://dx.doi.org/10.1136/ jnnp-2018-318483)
\end{abstract}

${ }^{1}$ Stroke Research Centre, Department of Brain Repair and Rehabilitation, UCL, London, UK ${ }^{2}$ The National Hospital for Neurology and Neurosurgery, Queen Square, London, UK ${ }^{3}$ Department of Statistical Science, UCL, London, UK

\section{Correspondence to} Dr David J Werring, The National Hospital for Neurology and Neurosurgery, UCL Institute of Neurology, London WC1N 3BG, UK; d.werring@ucl.ac.uk

Received 23 March 2018 Revised 21 June 2018

Accepted 18 July 2018 Published Online First 27 August 2018

(C) Author(s) (or their employer(s)) 2019. No commercial re-use. See rights and permissions. Published by BMJ.

To cite: Ziff OJ, Banerjee G, Ambler G, et al. J Neurol

Neurosurg Psychiatry

2019:90:75-83

\section{ABSTRACT}

Objective Whether statins increase the risk of intracerebral haemorrhage $(\mathrm{ICH})$ in patients with a previous stroke remains uncertain. This study addresses the evidence of statin therapy on $\mathrm{ICH}$ and other clinical outcomes in patients with previous ischaemic stroke (IS) or ICH.

Methods A systematic literature review and metaanalysis was performed in conformity with the Preferred Reporting Items for Systematic Reviews and Meta-Analyses guidelines to assess observational and randomised studies comparing statin therapy with control (placebo or no treatment) in patients with a previous ICH or IS. The risk ratios (RR) for the primary outcome (ICH) and secondary outcomes (IS, any stroke, mortality and function) were pooled using random effects meta-analysis according to stroke subtype. Results Forty-three studies with a combined total of 317291 patient-years of follow-up were included. In patients with previous ICH, statins had no significant impact on the pooled RR for recurrent ICH $(1.04,95 \%$ Cl 0.86 to $1.25 ; n=23695)$; however, statins were associated with significant reductions in mortality (RR 0.49, 95\% Cl 0.36 to $0.67 ; n=89$ 976) and poor functional outcome (RR $0.71,95 \% \mathrm{Cl} 0.67$ to 0.75 ; $\mathrm{n}=9113$ ). In patients with previous IS, statins were associated with a non-significant increase in ICH (RR $1.36,95 \% \mathrm{Cl} 0.96$ to $1.91 ; n=103525)$, but significantly lower risks of recurrent IS (RR $0.74,95 \% \mathrm{Cl} 0.66$ to $0.83 ; n=53162$ ), any stroke ( $R R \quad 0.82,95 \% \mathrm{Cl} 0.67$ to $0.99 ; n=55260$ ), mortality (RR $0.68,95 \% \mathrm{Cl} 0.50$ to $0.92 ; n=74648$ ) and poor functional outcome (RR 0.83, $95 \% \mathrm{Cl} 0.76$ to $0.91 ; n=34700$ ).

Conclusions Irrespective of stroke subtype, there were non-significant trends towards future ICH with statins. However, this risk was overshadowed by substantial and significant improvements in mortality and functional outcome among statin users.

Trial registration number CRD42017079863.

\section{INTRODUCTION}

Statin therapy has routinely been used to inhibit cholesterol synthesis and avoid cardiovascular events throughout the last three decades. They are recommended by both American and European guidelines to reduce risk of stroke and cardiovascular events in patients with cerebrovascular disease. ${ }^{12}$ Despite the demonstrated beneficial effects of statins in preventing first ever stroke, prescriptions remain suboptimal with age, gender, racial and geographic discrepancies. ${ }^{3}$ This may partially be explained by concerns around the potential risk of intracerebral haemorrhage $(\mathrm{ICH})$ with statins due to their antiplatelet and anticoagulant effects, particularly in patients with a previous $\mathrm{ICH}{ }^{45}$

In two large randomised trials, Stroke Prevention by Aggressive Reduction in Cholesterol Levels $\left(\right.$ SPARCL) ${ }^{6}$ and Heart Protection Study (HPS), ${ }^{7}$ the benefit in reducing recurrent ischaemic stroke was offset in part by an increased risk of haemorrhagic stroke. A risk benefit decision analysis of statin therapy in patients with prior ICH concluded that statin avoidance should be considered following ICH particularly in those with lobar $\mathrm{ICH}^{8}{ }^{8}$ Conversely, two meta-analyses of randomised trials enrolling patients without prior stroke found no significant association between statins and ICH with significant reductions in all-stroke and all-cause mortality with statin therapy. ${ }^{9} 10$ Additionally, statin use after ICH was associated with early neurological improvement at 6 months. ${ }^{11}$ There is, therefore, a clear imperative to define the place of statins in the clinical management of patients with a previous stroke at future risk of ICH.

In view of the potential usefulness of statins in patients with a previous stroke, and in an attempt to settle the uncertainty over adverse clinical outcomes, we assessed the efficacy and safety of statins by comprehensively meta-analysing all available observational and experimental studies. We aim to build on the previous meta-analyses by focusing on studies in which patients had an established ischaemic or haemorrhagic stroke. We used metaregression techniques to evaluate the association of study characteristics with the risk of clinical outcomes.

\section{METHODS}

\section{Eligibility criteria and search strategy}

All studies comparing clinical outcomes in participants treated with statins and control (placebo or no treatment) were evaluated, regardless of study design. We excluded studies where statins were used for primary prevention or did not provide comparative outcomes. Studies assessing secondary prevention of cerebrovascular disease were included. The definitions of ICH and ischaemic stroke used by each individual study were accepted. A systematic review of MEDLINE (1960 to June 2017), EMBASE (1980 to June 2017) and the Cochrane Library (until June 2017 issue) was 
performed. The search strategy included keywords and MeSH terms relating to statins and $\mathrm{ICH}$, ischaemic stroke, death and functional outcomes. We manually searched reference lists of relevant studies, investigated registers of ongoing trials and included studies after discussion with content experts. The review was conducted according to the Preferred Reporting Items for Systematic Reviews and Meta-Analyses guidelines and was prospectively registered with the PROSPERO database of systematic reviews (CRD42017079863). ${ }^{12}$

\section{Data collection, synthesis and risk of bias}

Two investigators (OJZ and GB) independently extracted and tabulated data in a standardised data extraction form. Discrepancies and missing data were resolved by group discussion, reference to the original publication and additional independent adjudication (DJW). All data were extracted from studies, including crude outcomes and adjusted analyses (multivariate adjustment and propensity matched). Careful note was made of the analysis method (including risk ratio (RR; preferred), OR or HR) and the population studied. Risk of bias was assessed with the Cochrane Collaboration's Risk of Bias Tool for Randomised Controlled Trials (RCT) and the Risk of Bias Assessment Tool for Non-randomized Studies (RoBANS), which address key criteria including selection bias, exposure measurement, blinding and selectivity of reporting. Assessment of bias risk was performed independently from data extraction, with each study assessed by two authors.

\section{Primary and secondary outcomes}

The predefined primary outcome was ICH. Secondary outcomes included ischaemic stroke, any stroke, all-cause mortality and poor functional outcome. The definitions used by each individual study were accepted. To investigate whether treatment effects vary between stroke types, analyses were subgrouped by previous ICH and previous ischaemic stroke.

\section{Statistical analysis}

Baseline demographics in the statin and control groups were compared using meta-analysis and summarised as the OR. Random effects meta-analysis was prespecified to combine estimates from different studies. Pooled binary event data for statin and control cohorts were compared using an RR with associated 95\%CIs using the method of DerSimonian and Laird. In cases where the OR was described, these were converted to RR $\left(\mathrm{RR}=\mathrm{OR} /\left([1-\mathrm{pRef}]+\left[\mathrm{pRef}{ }^{*} \mathrm{OR}\right]\right)\right.$, where pRef is the prevalence of the outcome in the reference group). RR and corresponding CI were log-transformed before pooling. HRs were included in the systematic review but not meta-analysis due to a scarcity of results presented in this way.

Where studies reported several results for the same outcome, we extracted the result based on the longest follow-up duration and most adjustment factors. Sensitivity analyses were performed according to study design (randomised trials and observational cohorts). The degree of heterogeneity between studies was quantitatively assessed using the $\mathrm{I}^{2}$ statistic $\left(\mathrm{I}^{2}\right.$ of $\geq 50 \%$ indicates substantial heterogeneity, $\geq 75 \%$ suggests considerable heterogeneity). Metaregression was performed to quantify the heterogeneity, assess the impact of baseline variables and risk of bias on estimates of each outcome, according to stroke subtype. Publication bias was evaluated by inspection of funnel plots and quantitatively assessed using Begg's and Egger's tests to identify small-study effects. A p value $<0.05$ was considered statistically
Table 1 Pooled weighted characteristics

\begin{tabular}{llll}
\hline & \multicolumn{2}{l}{ Statinversus control arm $(95 \% \mathrm{Cl})$} & \\
\cline { 2 - 3 } Baseline characteristic & OR & & P values \\
\hline Male gender & 1.08 & $(0.98$ to 1.18$)$ & 0.11 \\
\hline Diabetes & 1.49 & $(1.31$ to 1.69$)$ & $<0.001$ \\
AF & 1.00 & $(0.88$ to 1.14$)$ & 1.00 \\
\hline Smoker & 0.90 & $(0.73$ to 1.12$)$ & 0.34 \\
\hline Hypertension & 1.54 & $(1.25$ to 1.91$)$ & $<0.001$ \\
\hline Hyperlipidaemia & 4.32 & $(2.29$ to 8.13$)$ & $<0.001$ \\
\hline CAD & 2.05 & $(1.53$ to 2.74$)$ & $<0.001$ \\
\hline Anticoagulant & 1.71 & $(1.29$ to 2.26$)$ & $<0.001$ \\
Antiplatelet & 2.36 & $(1.69$ to 3.30$)$ & $<0.001$ \\
\hline
\end{tabular}

Meta-analysis of baseline demographics comparing statin-treated patients with control. $A F$, atrial fibrillation; $C A D$, coronary artery disease.

significant. Analyses were performed using STATA V.13.1 (StataCorp, Texas).

\section{RESULTS}

The search strategy identified 51 studies for systematic review, including 1324450 patients on statin therapy or control (placebo or no treatment) and 4098285 patient-years of follow-up (online supplementary efigure 1 ). Of the 51 studies, 36 were observational, ${ }^{11}{ }^{13-46}$ and 15 were randomised on the basis of statin therapy. ${ }^{674-59}$ Study descriptors are summarised in online supplementary etable 1 . Forty-three studies were suitable for inclusion in the quantitative meta-analysis comparing statins with control in patients with a previous stroke. Of the 43 studies, 15 provided data on patients with a previous $\mathrm{ICH}^{611} 17$ 34-36 38-44 47 48 and 29 reported outcomes in patients with a prior ischaemic stroke. ${ }^{13-15}{ }^{17-32} 45{ }^{4649-56}$ A total of 84 356 patients were taking statins (47.1\%) compared with 94597 in the control arms (52.8\%). The weighted average length of follow-up was 1.77 years with a range of 0.1-7.0.

Differences in key characteristics between statin and control groups are summarised in table 1 (for full baseline demographics, see online supplementary etable 2 ). Patients receiving statins had more diabetes, hypertension, hyperlipidaemia and coronary artery disease than controls and were more often receiving anticoagulant and antiplatelet drugs.

Meta-analysis was performed for five outcomes: ICH, ischaemic stroke, any stroke, all-cause mortality and functional outcome. A summary of the individual meta-analyses performed is presented in figure 1 and detailed results are discussed below. The risk of bias in individual studies is presented in online supplementary etables 3 and 4 . As expected, this was proportional to the robustness of study design, with RCTs having the lowest risk of bias. There was no evidence of small-study effects or publication bias in any of the outcomes assessed (all Egger's $\mathrm{p}>0.1)$

\section{Population: previous ICH}

Fifteen studies of patients with previous ICH were suitable for meta-analysis ( $\mathrm{n}=50$ 374; table 2; figure 2). ${ }^{611} 1734-3638-444748$

\section{Outcome: recurrent $\mathrm{ICH}$}

One randomised and two observational studies of statins in patients with previous ICH reporting the outcome recurrent ICH were included $(n=23695) .{ }^{34447}$ There was no difference in recurrent ICH between patients on statin and control (RR $1.04,95 \%$ CI 0.86 to $1.25, \mathrm{p}=0.70$ ). Two additional studies providing HRs were both concordant with this neutral result. ${ }^{3337}$ 


\section{Outcome}

Population: Previous intracerebral haemorrhage

Recurrent ICH (3)

All-cause mortality (12)

Poor functional outcome (7)

Population: Previous

ischaemic stroke

ICH (11)

Recurent ischaemic stroke (3)

Any stroke (7)

All-cause mortality (13)

Poor functional outcome (19)
No of events/total Statin group Control group

$2489 / 20643$
$6840 / 26778$
$2795 / 6889$

$258 / 26949$
$862 / 11492$
$1167 / 12353$
$1638 / 20251$
$3592 / 9902$

2915/9726
Risk ratio $(95 \% \mathrm{Cl})$
Risk ratio
$(95 \% \mathrm{Cl})$

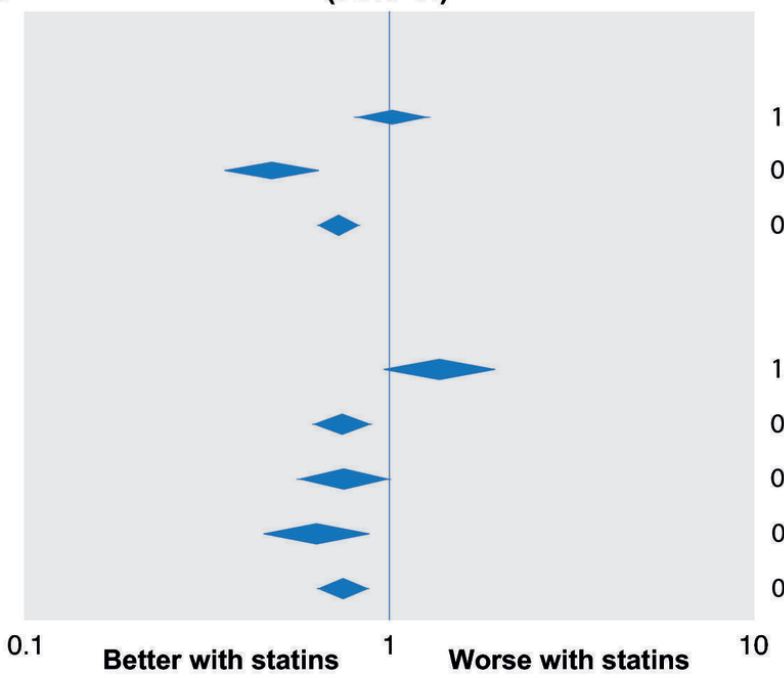

1.04 (0.86 to 1.25$)$

0.49 (0.36 to 0.67$)$

0.71 (0.67 to 0.75 )

1.36 (0.96 to 1.91$)$

0.74 (0.66 to 0.83 )

0.82 (0.67 to 0.99 )

0.68 (0.50 to 0.92 )

0.83 (0.76 to 0.91 )

Better with statins

Worse with statins

Figure 1 Summary of meta-analyses in observational and randomised studies on safety and efficacy of statins in patients with previous stroke (total studies). See figures 2 and 3 for study-level results. ICH, intracerebral haemorrhage.

Sensitivity analysis of study design demonstrated that although there was no significant difference between the randomised and observational studies, the observational studies had a noteworthy lower pooled relative risk than the randomised SPARCL subgroup (RR 1.02, 95\% CI 0.89 to 1.16 vs RR 3.73, 95\% CI 0.83 to 17.0 ; see figure 1 , online supplementary etable 5).

Outcome:ischaemic stroke

One substudy of statins in patients with previous ICH $(n=93)$ reported a non-significant increase in incident ischaemic stroke (RR $1.60,95 \%$ CI 0.28 to 9.14$){ }^{47}$

Outcome: any stroke

One substudy of statins in patients with previous ICH $(n=93)$ reported a non-significant increase in any stroke (RR 2.67, 95\% CI 0.90 to $7.90, \mathrm{p}=0.08){ }^{47}$

Outcome: all-cause mortality

In 15 studies with previous ICH $(n=89$ 976) there was a significant reduction in all-cause mortality with statins versus control
(RR $0.49,95 \%$ CI 0.36 to $0.67, \mathrm{p}<0.001$ ) but with significant heterogeneity $\left(\mathrm{I}^{2}=91.7 \%, \mathrm{p}<0.001\right) .{ }^{611} 1734-3638-414348$ Two additional studies reporting HRs were consistent with this finding. ${ }^{3342}$ Sensitivity analysis of study design demonstrated that observational studies had a lower pooled relative risk than the randomised analyses (RR $0.46,95 \%$ CI 0.33 to 0.63 vs RR 1.03 , 95\% CI 0.47 to 2.49; see online supplementary etable 5).

We performed an exploratory metaregression of the impact of differences in key baseline characteristics on all-cause mortality between statin and control patients. This analysis demonstrated that the mortality of benefit of statins was diminished when the statin group was composed of a smaller proportion of men than the control cohort $(p=0.003$; online supplementary etable 5). Additionally a greater mortality benefit with statins was associated with a more recent year of publication $(p=0.01)$.

\section{Outcome: poor functional outcome}

Seven studies of patients with previous ICH reported functional outcome $(n=9113)$, demonstrating a significant reduction in

Table 2 Summary of studies and patients according to cerebrovascular disease subtype

\begin{tabular}{|c|c|c|c|c|c|c|}
\hline Population & Outcome & Studies (n) & Statin patients & Control patients & Patients (n) & $\begin{array}{l}\text { Patient-years of } \\
\text { follow-up }\end{array}$ \\
\hline Systematic review & - & 51 & - & - & 1324450 & 4098285 \\
\hline Meta-analysis & - & 43 & 84356 & 94597 & 178953 & 317291 \\
\hline \multirow{6}{*}{$\begin{array}{l}\text { Previous intracerebral } \\
\text { haemorrhage }\end{array}$} & Total & 15 & 7645 & 42729 & 50374 & 91467 \\
\hline & Recurrent ICH & 3 & 3052 & 20643 & 23695 & 62930 \\
\hline & Ischaemic stroke & 1 & 45 & 48 & 93 & 456 \\
\hline & Any stroke & 1 & 45 & 48 & 93 & 456 \\
\hline & All-cause mortality & 15 & 57189 & 32787 & 89976 & 87954 \\
\hline & Poor functional outcome & 7 & 1942 & 7171 & 9113 & 5915 \\
\hline \multirow[t]{6}{*}{ Previous ischaemic stroke } & Total & 29 & 76711 & 51868 & 128579 & 225824 \\
\hline & $\mathrm{ICH}$ & 11 & 64005 & 39520 & 103525 & 210509 \\
\hline & Recurrent ischaemic stroke & 3 & 40808 & 12354 & 53162 & 50870 \\
\hline & Any stroke & 7 & 41643 & 13617 & 55260 & 59078 \\
\hline & All-cause mortality & 13 & 51500 & 23148 & 74648 & 43568 \\
\hline & Poor functional outcome & 19 & 15858 & 18842 & 34700 & 31907 \\
\hline
\end{tabular}




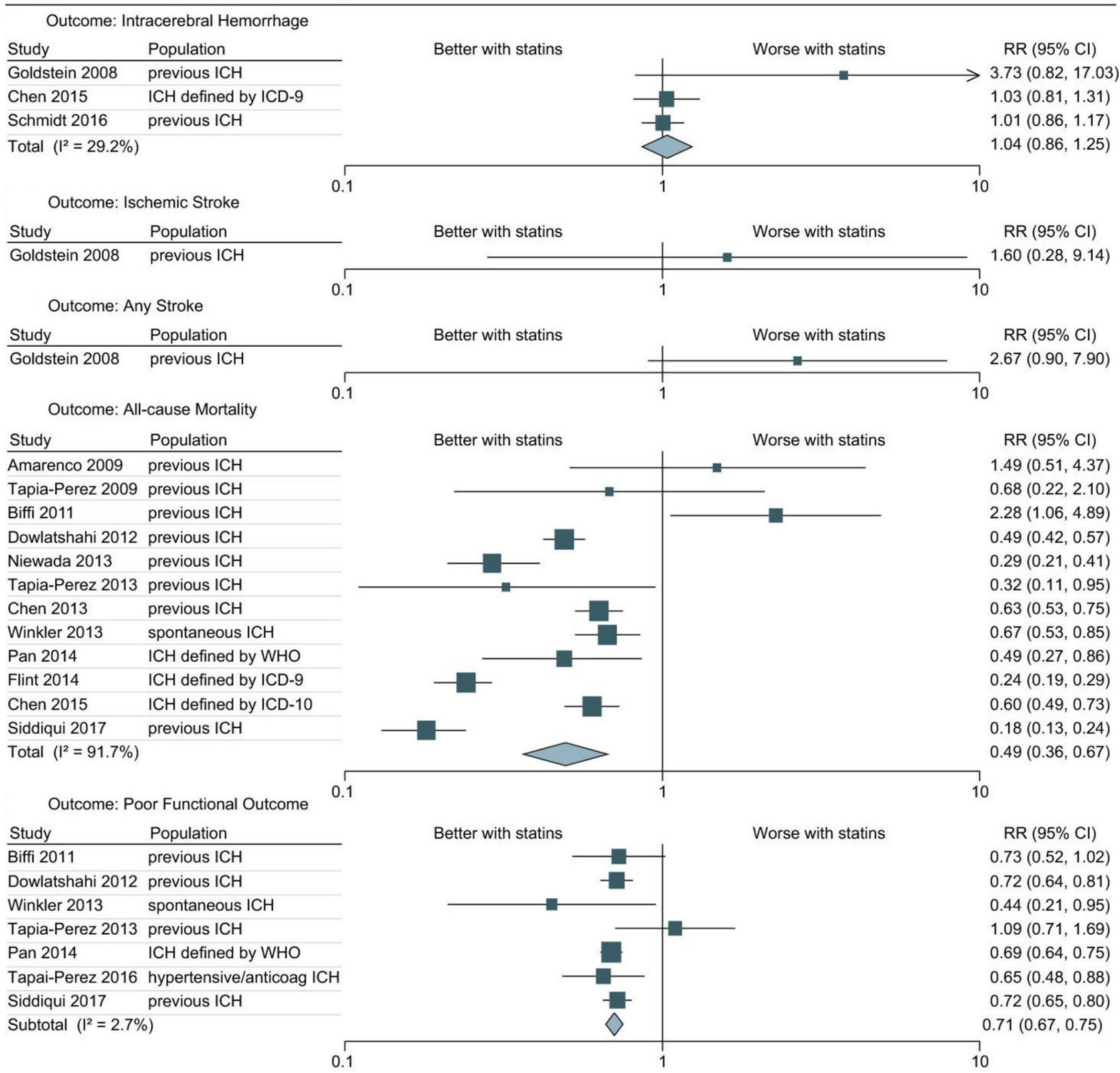

Figure 2 Forest plot of studies on association between statins and clinical outcomes in patients with previous intracerebral haemorrhage. The diamond represents the pooled difference using a random effects model. $I^{2}$ is the percentage of total variation across studies due to heterogeneity. Egger's test of small-study effects: $\mathrm{ICH} p=0.16$; all-cause mortality $p=0.88$; poor functional outcome $p=0.58$. Poor functional outcome was defined as modified Rankin Scale $(\mathrm{mRS})>2$ by all studies, except those by Dowlatshahi et $a^{36}$ which defined as mRS $>3$, Tapia-Perez et al ${ }^{11} 42$ which defined as National Institutes of Health Stroke Scale (NIHSS) > 15 and Winkler et a/ ${ }^{43}$ which defined as modified Barthel Index (mBI) <15. ICD, International Classification of Diseases; ICH, intracerebral haemorrhage; RR, risk ratio.

poor functional outcome among statin users compared with control (RR $0.71,95 \%$ CI 0.67 to $0.75, \mathrm{p}<0.001) .{ }^{11} 173640-43$

\section{Population: previous ischaemic stroke}

Twenty-nine studies of patients with previous ischaemic stroke were suitable for meta-analysis $(n=128$ 579; table 2; figure 3). ${ }^{13-15}$ 17-32 $454649-56$

Outcome: ICH

Eleven studies of statins in patients with prior ischaemic stroke reporting ICH were included $(n=103525){ }^{15} 18-21232629455152$ There was a non-significant increase in ICH with statins compared with control (RR 1.36, 95\% CI 0.96 to $1.91 ; \mathrm{p}=0.08$ ) but with substantial heterogeneity $\left(\mathrm{I}^{2}=79.3 ; \mathrm{p}<0.001\right)$. In studies that enrolled only patients with an ischaemic stroke undergoing thrombolysis for ischaemic stroke there was a non-significant increase in ICH with statins (RR $1.61,95 \%$ CI 0.77 to $3.34 ; p=0.20$ ) compared with the remaining six studies without thrombolysis (RR 1.21, 95\% CI 0.83 to 1.76 ; p=0.33). ${ }^{1518192945}$ Sensitivity analysis of study design demonstrated that observational studies had a lower pooled relative risk than the randomised trials (RR 1.73, 95\% CI 1.20 to 2.49 vs RR 1.28 , 95\% CI 0.85 to 1.93 ; see online supplementary etable 5).

An exploratory metaregression of the effect of study-level bias demonstrated that studies with lower bias reported a greater 


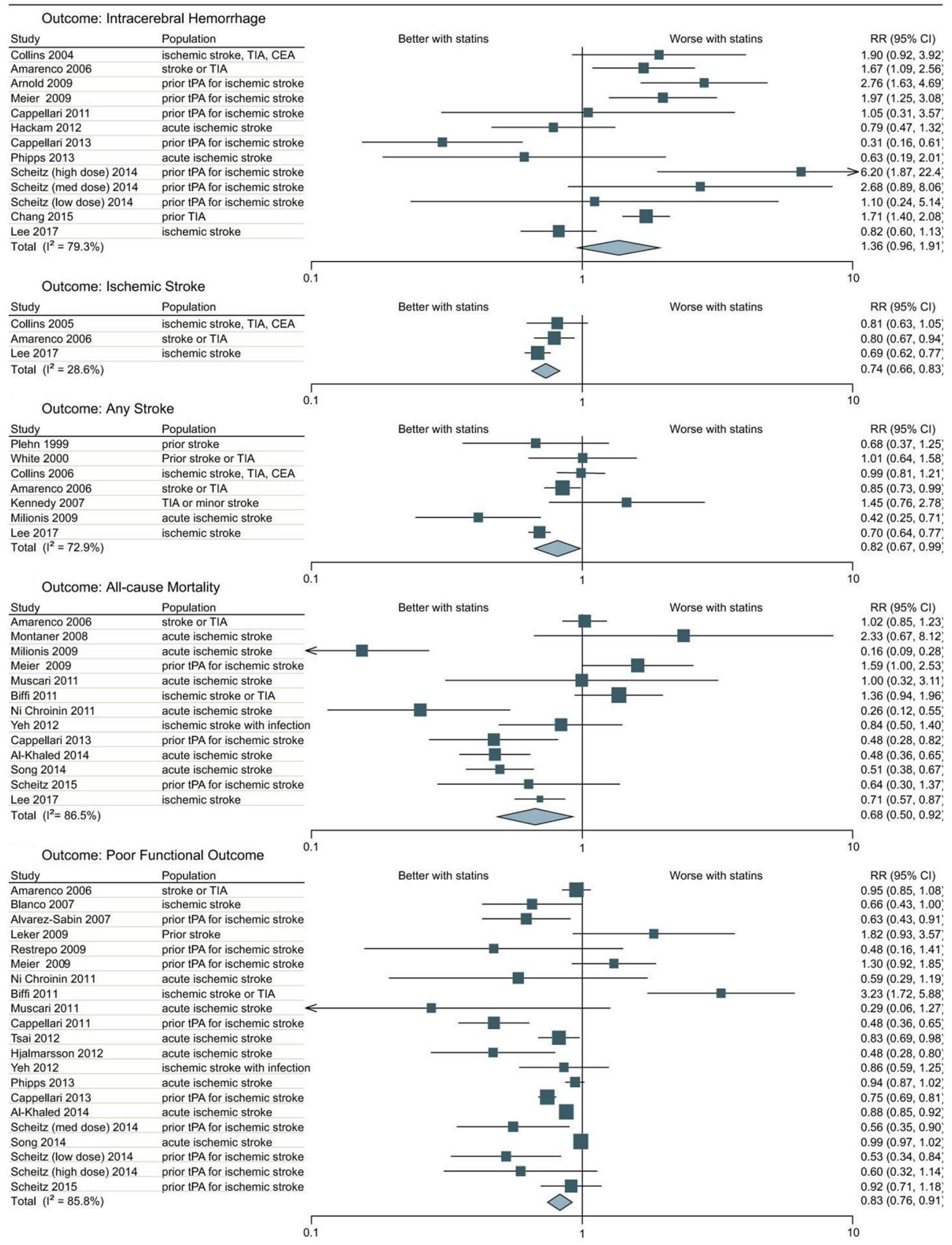

Figure 3 Forest plot of studies on association between statins and clinical outcomes in patients with previous ischaemic stroke. The diamond represents the pooled difference using a random effects model. $I^{2}$ is the percentage of total variation across studies due to heterogeneity. Egger's test of small-study effects: ICH $p=0.78$; ischaemic stroke $p=0.30$; any stroke $p=0.52$; all-cause mortality $p=0.54$; poor functional outcome $p=0.69$. Poor functional outcome was defined as modified Rankin Scale (mRS) $>2$ by all studies, except those by Alvarez-Sabin et $a^{14}$ and Song et a ${ }^{\beta 0}$ which defined as being dependent, and Leker et $a{ }^{24}$ as $m R S>3$. CEA, carotid endarterectomy; RR, risk ratio; TIA, transient ischaemic attack; tPA, tissue plasminogen activator.

association of statins with ICH $(\mathrm{p}=0.017$; online supplementary efigure 2). This was supported by a sensitivity analysis of study design, where the pooled two randomised trials reported a significant increase in ICH (RR 1.73, 95\% CI 1.20 to 2.49; $p=0.004)$, while the nine observational studies reported a neutral association (RR $1.28,95 \%$ CI 0.85 to $1.93 ; \mathrm{p}=0.23$ ). Metaregression was used to explore the impact of differences in key baseline characteristics between statin and control patients on ICH. This revealed that studies with a similar incidence of hyperlipidaemia in both the statin and control groups were associated with increased ICH with statins $(p=0.002)$. Conversely, studies where patients had a higher incidence of hyperlipidaemia in the statin group compared with control were associated with reduced ICH with statins. Additionally, studies with larger proportion of men in the statin arm were more likely to report an increase in $\mathrm{ICH}$ with statins $(p=0.025)$

Outcome: recurrent ischaemic stroke

Three studies of patients with previous ischaemic stroke $(n=53$ 162) revealed a reduction in recurrent ischaemic stroke with statins compared with control (RR $0.74,95 \%$ CI 0.66 to 0.83 ; $\mathrm{p}<0.001) .^{235152}$ Two additional studies reporting HRs were both consistent with this outcome. ${ }^{2160}$ 
Outcome: any stroke

Seven studies with previous ischaemic stroke were included ( $n=55260) .^{23} 4649-5254$ There was a borderline significant reduction in any stroke with statins compared with control (RR 0.82, $95 \%$ CI 0.67 to $0.99 ; \mathrm{p}=0.04$ ) but with significant heterogeneity $\left(\mathrm{I}^{2}=72.9 ; \mathrm{p}=0.001\right)$.

\section{Outcome: all-cause mortality}

In 13 studies pertaining to the prior ischaemic stroke cohort $(\mathrm{n}=74$ 648), there was a reduction in all-cause mortality with statins compared with control (RR $0.68,95 \%$ CI 0.50 to 0.92 , $\mathrm{p}=0.01)^{13171823252930324546525556}$ but with considerable heterogeneity $\left(\mathrm{I}^{2}=86.5 ; \mathrm{p}<0.001\right)$. Two additional analyses reporting HRs both demonstrated significant reductions in mortality. ${ }^{16} 22$ Sensitivity analysis of study design demonstrated that although there was a significant reduction in mortality in the pooled observational studies (RR $0.60,95 \%$ CI 0.42 to 0.85 ) there was no statistical difference in the pooled randomised analyses (RR $1.04,95 \%$ CI 0.87 to 1.24 ; online supplementary etable 5).

\section{Outcome: poor functional outcome}

Twenty-one analyses $(n=34700)$ in 19 studies of patients with prior ischaemic stroke reported functional outcome. Together these demonstrated that statin use was significantly associated with a reduction in poor functional outcome compared with control (RR $0.83,95 \%$ CI 0.76 to $0.91, p<0.001$ ) although with significant heterogeneity $\left(\mathrm{I}^{2}=85.8 ; \mathrm{p}<0.001\right) .{ }^{13} 1417-192224-3245525356$

\section{DISCUSSION}

In our comprehensive meta-analysis including a combined total of over 300000 patient-years of follow-up we found that in patients with a previous $\mathrm{ICH}$, statins were not associated with an increased risk of recurrent ICH. In patients with previous ischaemic stroke, we found a clear benefit of statins in reducing recurrent ischaemic stroke at the expense of a non-significant increase in ICH. Statins were associated with substantial and significant improvements in mortality and functional outcome irrespective of stroke subtype.

\section{Previous ICH}

Statins exert beneficial cardiovascular pleotropic effects on endothelial dysfunction through normalising vasomotion, increasing bioavailability of nitric oxide and supressing inflammatory responses. ${ }^{61}$ However, the antiplatelet and anticoagulant effects of statins have raised concerns that they may increase the risk of ICH. Statins have also been hypothesised to have potentially harmful consequences in acute ICH where their diverse pharmacological properties may contribute to haematoma expansion. ${ }^{62}$ In both the HPS and SPARCL trials, which enrolled patients with a previous stroke, statins were associated with increased ICH compared with placebo. ${ }^{75}$ Of these two trials, only SPARCL provided a subgroup analysis of patients with a previous haemorrhagic stroke, demonstrating a non-significantly increased risk of recurrent $\mathrm{ICH} .{ }^{47}$ In contrast, four larger observational cohort studies all demonstrated a neutral effect of statins on recurrent $\mathrm{ICH},{ }^{33} 343744$ consistent with our findings.

We found that in ICH survivors statins were associated with improved mortality and functional outcome with no significant effect on recurrent $\mathrm{ICH}$. We were unable to meta-analyse the outcomes ischaemic stroke and any stroke type as the only data available were from the haemorrhagic stroke subgroup population of the SPARCL trial $(n=93) .{ }^{47}$ These results do not support withholding statins after ICH, but large RCTs are still needed to consolidate these findings.

Most ICHs are due to cerebral small vessel disease hypertensive arteriopathy (arteriolosclerosis), which affects deep perforating vessels, and cerebral amyloid angiopathy (CAA), which affects superficial cortical and leptomeningeal vessels. Thus, while hypertension is the strongest risk factor for deep ICH, a substantial proportion of lobar ICH is due to CAA. CAA has a high recurrence risk ( $7.4 \%$ per year) in a pooled analysis of cohort studies $^{63}$ so has caused the strongest concerns regarding statin use. Observational and randomised data suggest that recurrent $\mathrm{ICH}$ can be reduced by antihypertensive therapy ${ }^{64}$; however, CAA currently lacks any specific preventative therapy. A retrospective analysis found that statins in patients with $\mathrm{ICH}$ were associated with microbleeds on MRI, particularly of cortico-subcortical distribution, commonly observed in CAA. ${ }^{65}$ Thus, although our findings are reassuring, we were unable to stratify by ICH location or presumed cause, so decisions in ICH survivors require an individualised patient assessment of indication, comorbidity and the goal of statin therapy. Unsurprisingly, among stroke physicians the use of statins in patients following ICH remains contentious. American guidelines recommend statins in patients with ICH due to insufficient data to advise restriction (class IIb; level C) while European guidelines do not address the issue. ${ }^{12}$ Unfortunately, the only double-blinded placebo controlled RCT of statins in patients with ICH (NCT00718328) terminated early due to poor recruitment.

\section{Previous ischaemic stroke}

In survivors of ischaemic stroke, statins were associated with substantial and significant improvements in mortality, functional outcome and ischaemic stroke, with a non-significant trend towards increased ICH. Although epidemiological data indicate a modest link between high serum low-density lipoprotein (LDL) and greater risk of ischaemic stroke, they have also pointed towards an association of low LDL and a heightened risk of $\mathrm{ICH}^{6}{ }^{66}$ By reducing serum cholesterol, statins may reduce the integrity of the vasculature leading to arterial necrosis and microaneurysm formation. ${ }^{67} \mathrm{~A}$ previous meta-analysis of randomised trials of statins for primary and secondary prevention of stroke demonstrated significant reductions in LDL and ischaemic stroke risk in both primary and secondary prevention, but a significant increase in ICH was identified in secondary prevention trials. ${ }^{68}$ This finding was largely based on the only dedicated secondary prevention trial of stroke, SPARCL, which identified a significant reduction in recurrent ischaemic stroke but with a higher incidence of $\mathrm{ICH}^{59}$ Similarly in the HPS trial previous stroke subgroup, there was a 91\% increased risk in haemorrhagic stroke with statins. ${ }^{7}$ We found that when these trials are combined with observational studies and limited to secondary prevention, this ICH risk persists, although non-significantly.

Nevertheless, given the potentially increased risk of ICH with statin treatment, physicians should have caution in recommending statins to individuals with risk factors for ICH. Indeed, we found substantial heterogeneity in treatment effect indicating a 'one size fits all' approach to statins may be inappropriate. For example, while the effectiveness of statin therapy in patients with previous ischaemic stroke due to atherosclerotic disease is clear, in those due to atrial fibrillation (AF) the evidence is less obvious. Indeed patients with stroke and AF, who were excluded from SPARCL, often have higher bleeding risks due to concomitant anticoagulation. ${ }^{52}$ Another important concomitant therapy to consider is thrombolysis, which further adds to the 
haemorrhagic transformation risk. ${ }^{45}$ The results of our sensitivity analysis confirmed that statins increased the risk of ICH in patients with ischaemic stroke treated with thrombolysis. Age is another important component of bleeding risk. Unfortunately numerous statin trials excluded frail elders casting doubt on how results might translate to those over 80 years old. Only with careful patient selection can an optimal balance between efficacy and safety be achieved.

If statins are considered in stroke survivors, then further contentious questions arise, including: (1) which statin; (2) what dose; (3) when to initiate; (4) and when to withdraw. With regard to agent and dose, the ongoing Treat Stroke to Target trial (NCT01252875) will provide clarity on targeted LDL levels and vascular events among survivors of ischaemic stroke. Regarding timing, surrogate marker studies indicate a role of statins in the acute phase of ischaemic stroke through upregulation of nitric oxide, fibrinolytic and antithrombotic mechanisms; however, the major statin trials typically did not enrol patients until $\sim 3$ months after stroke. ${ }^{52} 69$ The only randomised trial to investigate timing per se demonstrated no improvement in neurological function at 90 days with early ( $<24$ hours) versus delayed $(7$ days) therapy, although included patients had low stroke severity who may not have substantial disease substrate to benefit. ${ }^{70}$

As statins are often not prescribed until clinicians detect presence of cardiovascular disease risk factors, treatment with statins is likely to be influenced by the probability of $\mathrm{ICH}$, creating 'confounding by indication'. We have demonstrated differences in baseline characteristics between patients in statin and control groups in observational studies and exposed their impact on ICH through metaregression analysis. These differences may partly explain the conflicting results between randomised and observational studies ${ }^{2145}$ a problem not exclusive to stroke trials. ${ }^{71}$

Taking all studies into account, despite prescription biases the net effect of statin use appears clearly beneficial for mortality and functional outcome, even though an increased risk of ICH may partly offset these improvements. Our findings thus suggest that statins should continue to be considered in those with a previous stroke (including $\mathrm{ICH}$ ) to reduce mortality and improve functional outcome, but caution should be taken in individuals at high risk for ICH (eg, older anticoagulated patients with poorly controlled hypertension or CAA). In these patients alternative approaches to manage hyperlipidaemia should be considered, for instance through upregulation of LDL receptors using the novel PCSK9 inhibitors. ${ }^{72}$

\section{LIMITATIONS}

Our review is based on published data of independent studies, performed in accordance with explicit, reproducible methodology. While meta-analysis of individual patient data is ideal, it is unrealistic with such large data groupings across a wide number of studies. We recognise a number of drawbacks of our study. First, there is a deficiency in sample sizes from both randomised and observational studies to generate adequately powered pooled effect estimates especially in the previous ICH cohort. There were insufficient data to perform meta-analysis of statin dose, statin type or the impact of location of ICH (lobar vs deep). Second, definitions of ICH and ischaemic stroke between studies differed with potential for miscategorisation. Although some studies precisely reported the stroke aetiology, type and severity, many did not. Third, due to expected disparities in study designs and populations, we prespecified a random effects model. Indeed, we noted substantial heterogeneity in treatment effect for many of the assessed outcomes. However, most of the heterogeneity was caused by the effect magnitude instead of the effect direction. Finally, although no signal of publication bias was identified, statistical assessments can be misrepresentative particularly with considerable heterogeneity. ${ }^{73}$

\section{CONCLUSION}

In patients with $\mathrm{ICH}$, statins did not increase recurrent ICH. In survivors of ischaemic stroke, although statins substantially and significantly reduced recurrent ischaemic stroke, there was a non-significant increase in ICH. Nonetheless, statins show clear benefits in reducing mortality and improving functional outcome irrespective of stroke subtype. These results were predominantly based on observational data with insufficient randomised trial data available. Given that observational data are subject to inherent confounding, future randomised trials of statins in patients with cerebrovascular disease (especially $\mathrm{ICH}$ survivors) are required to clarify the safety of this therapy on future $\mathrm{ICH}$ risk.

Acknowledgements We thank Kate Brunskill, Deputy Librarian at the Queens Square Library, UCL Institute of Neurology, for her expertise and guidance in finalising the search strategy.

Contributor OJZ developed the eligibility criteria, performed the primary literature search, contributed to data extraction and drafting of the manuscript. GB contributed to data extraction and critical revision of the manuscript. GA contributed to statistical analysis and critical revision of the manuscript. DJW designed the study concept, led the study group and critically revised the manuscript.

Funding This work was undertaken at the University College London Hospitals NHS Foundation Trust/University College London who received a proportion of funding from the Department of Health's National Institute for Health Research Biomedical Research Centres funding scheme. OJZ is funded by the National Institute of Health Research UK. GB received research support from the Rosetrees Trust. DJW receives research funding support from the British Heart Foundation and the Stroke Association (TSA BHF 2009/01; TSA PPA 2015/04; BHF CS/17/6/33361).

Competing interests $0 J Z, G B$ and GA have no relevant conflicts. DJW was UK chief investigator for A9951024 (Pfizer) and has received consultancy and lecture fees from Bayer.

Patient consent Not required.

Provenance and peer review Not commissioned; externally peer reviewed. Data sharing statement No additional data are available.

\section{REFERENCES}

1 Hemphill JC, Greenberg SM, Anderson CS, et al. Guidelines for the Management of Spontaneous Intracerebral Hemorrhage: A Guideline for Healthcare Professionals From the American Heart Association/American Stroke Association. Stroke 2015;46:2032-60.

2 Steiner T, Al-Shahi Salman R, Beer R, et al. European Stroke Organisation (ESO) guidelines for the management of spontaneous intracerebral hemorrhage. Int J Stroke 2014;9:840-55.

3 Albright KC, Howard VJ, Howard G, et al. Age and sex disparities in discharge statin prescribing in the stroke belt: evidence from the reasons for geographic and racial differences in stroke study. J Am Heart Assoc 2017;6:e005523.

4 Hong KS, Lee JS. Statins in acute ischemic stroke: a systematic review. J Stroke 2015;17:282-301.

5 Cordenier A, De Smedt A, Brouns R, et al. Pre-stroke use of statins on stroke outcome: a meta-analysis of observational studies. Acta Neurol Belg 2011;111:261-7.

6 Amarenco P, Benavente O, Goldstein LB, et al. Results of the Stroke Prevention by Aggressive Reduction in Cholesterol Levels (SPARCL) trial by stroke subtypes. Stroke 2009:40:1405-9.

7 Heart Protection Study Collaborative Group. MRC/BHF Heart Protection Study of cholesterol lowering with simvastatin in 20,536 high-risk individuals: a randomised placebo-controlled trial. Lancet 2002;360:7-22.

8 Westover MB, Bianchi MT, Eckman MH, et al. Statin use following intracerebra hemorrhage: a decision analysis. Arch Neurol 2011;68:573-9.

9 McKinney JS, Kostis WJ. Statin therapy and the risk of intracerebral hemorrhage: a meta-analysis of 31 randomized controlled trials. Stroke 2012;43:2149-56.

10 Baigent C, Keech A, Kearney PM, et al. Efficacy and safety of cholesterol-lowering treatment: prospective meta-analysis of data from 90,056 participants in 14 randomised trials of statins. Lancet 2005;366:1267-78. 
11 Tapia-Pérez JH, Rupa R, Zilke R, et al. Continued statin therapy could improve the outcome after spontaneous intracerebral hemorrhage. Neurosurg Rev 2013;36:279-87.

12 ZiffOJ, BanerjeeG, AmblerA. The impact of statins on clinical outcomes in patients with a previous stroke: a systematic review and meta-analysis CRD42017079863. PROSPERO International prospective register of systematic reviews. 2017.

13 Al-Khaled M, Matthis C, Eggers J. Statin treatment in patients with acute ischemic stroke. Int J Stroke 2014;9:597-601.

14 Alvarez-Sabín J, Huertas R, Quintana M, et al. Prior statin use may be associated with improved stroke outcome after tissue plasminogen activator. Stroke 2007;38:1076-8.

15 Prior statin use, intracranial hemorrhage and outcome after intra-arterial thrombolysis for acute ischemic stroke. Stockholm, Sweden: 18th European Stroke Conference, 2009

16 Asberg S, Henriksson KM, Farahmand B, et al. Ischemic stroke and secondary prevention in clinical practice: a cohort study of 14,529 patients in the Swedish Stroke Register. Stroke 2010;41:1338-42.

17 Biffi A, Devan WJ, Anderson CD, et al. Statin use and outcome after intracerebra hemorrhage: case-control study and meta-analysis. Neurology 2011;76:1581-8.

18 Cappellari M, Bovi P, Moretto G, et al. The THRombolysis and STatins (THRaST) study. Neurology 2013;80:655-61.

19 Cappellari M, Deluca C, Tinazzi M, et al. Does statin in the acute phase of ischemic stroke improve outcome after intravenous thrombolysis? A retrospective study. J Neurol Sci 2011;308(1-2):128-34.

20 ChangCH, LinCH, CaffreyJL. Risk of intracranial hemorrhage from statin use in :A nationwide cohort Study. Circulation 2015;131:2070-8.

21 Hackam DG, Austin PC, Huang A, et al. Statins and intracerebral hemorrhage: a retrospective cohort study. Arch Neurol 2012;69:39-45

22 Hialmarsson C, Bokemark L, Manhem K, et al. The effect of statins on acute and long-term outcome after ischemic stroke in the elderly. Am J Geriatr Pharmacother 2012;10:313-22.

23 Lee M, Saver JL, Wu YL, et al. Utilization of Statins Beyond the Initial Period After Stroke and 1-Year Risk of Recurrent Stroke. J Am Heart Assoc 2017:6:e005658.

24 Leker RR, Khoury ST, Rafaeli G, et al. Prior use of statins improves outcome in patients with intracerebral hemorrhage: prospective data from the National Acute Stroke Israeli Surveys (NASIS). Stroke 2009:40:2581-4.

25 Ní Chróinín D, Callaly EL, Duggan J, et al. Association between acute statin therapy, survival, and improved functional outcome after ischemic stroke: the North Dublin Population Stroke Study. Stroke 2011;42:1021-9.

26 Phipps MS, Zeevi N, Staff I, et al. Stroke severity and outcomes for octogenarians receiving statins. Arch Gerontol Geriatr 2013;57:377-82.

27 Restrepo L, Bang OY, Ovbiagele B, et al. Impact of hyperlipidemia and statins on ischemic stroke outcomes after intra-arterial fibrinolysis and percutaneous mechanical embolectomy. Cerebrovasc Dis 2009;28:384-90.

28 Scheitz JF, Endres M, Heuschmann PU, et al. Reduced risk of poststroke pneumonia in thrombolyzed stroke patients with continued statin treatment. Int J Stroke 2015;10:61-6.

29 Scheitz JF, Seiffge DJ, Tütüncü S, et al. Dose-related effects of statins on symptomatic intracerebral hemorrhage and outcome after thrombolysis for ischemic stroke. Stroke 2014;45:509-14.

30 Song $B$, Wang Y, Zhao X, et al. Association between statin use and shortterm outcome based on severity of ischemic stroke: a cohort study. PLoS One 2014;9:e84389.

31 Tsai NW, Lee LH, Huang CR, et al. The association of statin therapy and highsensitivity C-reactive protein level for predicting clinical outcome in acute noncardioembolic ischemic stroke. Clin Chim Acta 2012;413(23-24):1861-5.

32 Yeh PS, Lin HJ, Chen PS, et al. Effect of statin treatment on three-month outcomes in patients with stroke-associated infection: a prospective cohort study. Eur J Neurol 2012;19:689-95.

33 Statin therapy and the risk of death and recurrent intracerebral hemorrhage. International Journal of Stroke Conference: European Stroke Organisation Annual Conference, Glasgow United Kingdom. 2015.

34 Chen PS, Cheng CL, Chang YC, et al. Early statin therapy in patients with acute intracerebral hemorrhage without prior statin use. Eur J Neurol 2015;22:773-80.

35 Early statin therapy reduces all-cause mortality in acute hemorrhagic stroke patients without prior statin use. European Society of Cardiology, ESC Congress 2013. Amsterdam, The Netherlands: European Heart Journal, 2013.

36 Dowlatshahi D, Demchuk AM, Fang J, et al. Association of statins and statin discontinuation with poor outcome and survival after intracerebral hemorrhage. Stroke 2012;43:1518-23

37 FitzMaurice $E$, Wendell $L$, Snider $R$, et al. Effect of statins on intracerebral hemorrhage outcome and recurrence. Stroke 2008;39:2151-4.

38 Flint $\mathrm{AC}$, Conell C, Rao VA, et al. Effect of statin use during hospitalization for intracerebral hemorrhage on mortality and discharge disposition. JAMA Neurol 2014;71:1364-71.

39 Niewada M, Skowrońska M, Sarzyńska-Długosz IM, et al. Pharmacotherapy prior to and in acute haemorrhagic stroke. The use of pharmacotherapy and drugs associated outcomes in real-world practice - findings from the Polish Hospital Stroke Registry. Neurol Neurochir Pol 2013;47:517-24.
40 Pan YS, Jing J, Wang YL, et al. Use of statin during hospitalization improves the outcome after intracerebral hemorrhage. CNS Neurosci Ther 2014;20:548-55.

41 Siddiqui FM, Langefeld CD, Moomaw CJ, et al. Use of Statins and Outcomes in Intracerebral Hemorrhage Patients. Stroke 2017:48:2098-104.

42 Tapia-Perez JH, Zilke R, Schneider T. Match-study of statin therapy in spontaneous intracerebral hemorrhage: is the discontinuation reasonable? J Neurosurg Sci 2016:60:301-12

43 Winkler J, Shoup JP, Czap A, et al. Long-term improvement in outcome after intracerebral hemorrhage in patients treated with statins. J Stroke Cerebrovasc Dis 2013;22:e541-e545.

44 Schmidt LB, Goertz S, Wohlfahrt J, et al. Recurrent Intracerebral Hemorrhage: Associations with Comorbidities and Medicine with Antithrombotic Effects. PLoS One 2016:11:e0166223.

45 Meier N, Nedeltchev K, Brekenfeld C, et al. Prior statin use, intracranial hemorrhage, and outcome after intra-arterial thrombolysis for acute ischemic stroke. Stroke 2009;40:1729-37

46 Milionis HJ, Giannopoulos S, Kosmidou M, et al. Statin therapy after first stroke reduces 10-year stroke recurrence and improves survival. Neurology 2009;72:1816-22

47 Goldstein LB, Amarenco P, Szarek M, et al. Hemorrhagic stroke in the Stroke Prevention by Aggressive Reduction in Cholesterol Levels study. Neurology 2008:70(24 Pt 2):2364-70.

48 Tapia-Perez H, Sanchez-Aguilar M, Torres-Corzo JG, et al. Use of statins for the treatment of spontaneous intracerebral hemorrhage: results of a pilot study. Cent Eur Neurosurg 2009;70:15-20.

49 Plehn JF, Davis BR, Sacks FM, et al. Reduction of stroke incidence after myocardia infarction with pravastatin: the Cholesterol and Recurrent Events (CARE) study. The Care Investigators. Circulation 1999;99:216-23.

50 White HD, Simes RJ, Anderson NE, et al. Pravastatin therapy and the risk of stroke. $N$ Engl J Med 2000;343:317-26.

51 Collins R, Armitage J, Parish S, et al. Effects of cholesterol-lowering with simvastatin on stroke and other major vascular events in 20536 people with cerebrovascular disease or other high-risk conditions. Lancet 2004;363:757-67.

52 Amarenco P, Bogousslavsky J, Callahan A. High-dose atorvastatin after stroke or transient ischemic attack. J Vasc Surg 2006:44:1374-59.

53 Blanco M, Nombela F, Castellanos M, et al. Statin treatment withdrawal in ischemic stroke: a controlled randomized study. Neurology 2007;69:904-10.

54 Kennedy J, Hill MD, Ryckborst KJ, et al. Fast assessment of stroke and transient ischaemic attack to prevent early recurrence (FASTER): a randomised controlled pilot trial. Lancet Neurol 2007;6:961-9.

55 Montaner J, Chacón P, Krupinski J, et al. Simvastatin in the acute phase of ischemic stroke: a safety and efficacy pilot trial. Eur J Neurol 2008;15:82-90.

56 Muscari A, Puddu GM, Santoro N, et al. The atorvastatin during ischemic stroke study: a pilot randomized controlled trial. Clin Neuropharmacol 2011;34:141-7.

57 Sacks FM, Pfeffer MA, Moye LA, et al. The effect of pravastatin on coronary events after myocardial infarction in patients with average cholesterol levels. Cholesterol and Recurrent Events Trial investigators. N Engl J Med 1996;335:1001-9.

58 Long-Term Intervention with Pravastatin in Ischaemic Disease (LIPID) Study Group. Prevention of cardiovascular events and death with pravastatin in patients with coronary heart disease and a broad range of initial cholesterol levels. N Eng/ J Med 1998;339:1349-57

59 Goldstein LB, Amarenco P, Zivin J, et al. Statin treatment and stroke outcome in the Stroke Prevention by Aggressive Reduction in Cholesterol Levels (SPARCL) trial. Stroke 2009:40:3526-31.

60 Uchiyama S, Ikeda Y, Urano Y, et al. The Japanese aggrenox (extended-release dipyridamole plus aspirin) stroke prevention versus aspirin programme (JASAP) study: a randomized, double-blind, controlled trial. Cerebrovasc Dis 2011;31:601-13

61 Davignon J. Beneficial cardiovascular pleiotropic effects of statins. Circulation 2004;109(23 Suppl 1):III39-43

62 Ural AU, Yilmaz MI, Avcu F, et al. Treatment with cerivastatin in primary mixed hyperlipidemia induces changes in platelet aggregation and coagulation system components. Int J Hematol 2002;76:279-83.

63 Charidimou A, Imaizumi T, Moulin S, et al. Brain hemorrhage recurrence, small vesse disease type, and cerebral microbleeds: A meta-analysis. Neurology 2017;89:820-9.

64 Biffi A, Anderson CD, Battey TW, et al. Association between blood pressure control and risk of recurrent intracerebral hemorrhage. JAMA 2015;314:904-12.

65 Haussen DC, Henninger N, Kumar S, et al. Statin use and microbleeds in patients with spontaneous intracerebral hemorrhage. Stroke 2012;43:2677-81.

66 Ebrahim S, Sung J, Song YM, et al. Serum cholesterol, haemorrhagic stroke, ischaemic stroke, and myocardial infarction: Korean national health system prospective cohort study. BMJ 2006;333:22.

67 Wang X, Dong Y, Qi X, et al. Cholesterol levels and risk of hemorrhagic stroke: a systematic review and meta-analysis. Stroke 2013;44:1833-9.

68 Amarenco P, Labreuche J. Lipid management in the prevention of stroke: review and updated meta-analysis of statins for stroke prevention. Lancet Neurol 2009;8:453-63.

69 Asahi M, Huang Z, Thomas S, et al. Protective effects of statins involving both eNOS and tPA in focal cerebral ischemia. J Cereb Blood Flow Metab 2005;25:722-9. 
70 International stroke conference. LB17 -Randomized controlled trial of early versus delayed statin therapy in patients with acute ischemic stroke. 2017.

71 Ziff OJ, Lane DA, Samra M, et al. Safety and efficacy of digoxin: systematic review and meta-analysis of observational and controlled trial data. BMJ 2015;351:h4451.
72 Castilla-Guerra L, Fernández-Moreno MC, Rico-Corral MA. Cholesterol and stroke: Roll of PCSK9 inhibitors. Neurologia 2017.

73 Terrin N, Schmid CH, Lau J. In an empirical evaluation of the funnel plot, researchers could not visually identify publication bias. J Clin Epidemiol 2005;58:894-901. 